\title{
VARIANTS OF THE KAPPA FUNCTION
}

\author{
Yuzen TANAKA
}

(Received 15 February 2006 and revised 25 June 2006)

\begin{abstract}
Kaneko and Yoshida introduced the kappa function $\kappa$ by the property $J(\kappa(z))=\lambda(z)$, where $J$ and $\lambda$ are the elliptic modular functions. This paper constructs several variants of the kappa function using other pairs of modular functions, and gives their explicit Fourier expansions.
\end{abstract}

\section{Introduction}

The Gauss hypergeometric differential equation with parameters $(a, b, c)$

$$
E(a, b, c): z(1-z) \frac{d^{2} u}{d z^{2}}+(c-(a+b+1) z) \frac{d u}{d z}-a b u=0
$$

has two linearly independent solutions. The Schwarz map $s: \mathbb{P}^{1}-\{0,1, \infty\} \longrightarrow \mathbb{P}^{1}$ is defined by the ratio of these two solutions.

The elliptic modular functions $J(z)$ and $\lambda(z)$ coincide with the inverse functions of the Schwarz maps of $E\left(\frac{1}{12}, \frac{5}{12}, 1\right)$ and $E\left(\frac{1}{2}, \frac{1}{2}, 1\right)$, respectively. They are related as

$$
J(z)=P(\lambda(z))=\frac{4}{27} \frac{\left(1 \lambda(z)+\lambda(z)^{2}\right)^{3}}{\lambda(z)^{2}(1-\lambda(z))^{2}} .
$$

On the other hand, Kaneko and Yoshida $[\mathbf{K Y}]$ introduced the kappa function $\kappa$ by the relation $\lambda(z)=J(\kappa(z))$; see Figure 1 .

In this paper we study several variants of the kappa function.

\section{Variants of the lambda function}

We recall some facts about the modular function.

2000 Mathematics Subject Classification: Primary 30C20, 30F35, 33C05.

Keywords and Phrases: covariant function; hypergeometric function; Schwarz's $s$-function; elliptic modular function. 


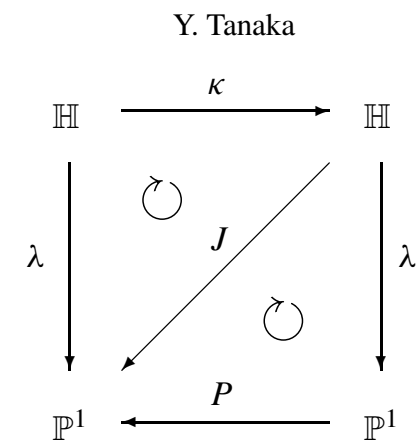

FIGURE 1. Relations among $\kappa, \lambda$ and $J$.

\subsection{Some triangle groups}

Define subgroups of $\Gamma=\operatorname{SL}(2, \mathbb{Z})$ as

$$
\begin{aligned}
& \Gamma(2)=\left\{\left(\begin{array}{ll}
a & b \\
c & d
\end{array}\right) \in \operatorname{SL}(2, \mathbb{Z}) \mid \begin{array}{l}
a \equiv d \equiv 1 \\
b \equiv c \equiv 0
\end{array}(\bmod 2)\right\} \\
& \Gamma_{1,2}=\left\{\left(\begin{array}{ll}
a & b \\
c & d
\end{array}\right) \in \operatorname{SL}(2, \mathbb{Z}) \mid a b \equiv c d \equiv 0(\bmod 2)\right\} .
\end{aligned}
$$

Setting

$$
\gamma_{i}=\left(\begin{array}{cc}
0 & -1 \\
1 & 0
\end{array}\right), \quad \gamma_{\rho_{2}}=\left(\begin{array}{cc}
1 & 1 \\
-1 & 0
\end{array}\right), \quad \gamma_{\infty}=\left(\begin{array}{ll}
1 & 2 \\
0 & 1
\end{array}\right), \quad \gamma_{0}=\left(\begin{array}{ll}
1 & 0 \\
2 & 1
\end{array}\right),
$$

we have

$$
\Gamma=\left\langle\gamma_{i}, \gamma_{\rho_{2}}\right\rangle, \quad \Gamma(2)=\left\langle\gamma_{\infty}, \gamma_{0}\right\rangle, \quad \Gamma_{1,2}=\left\langle\gamma_{i}, \gamma_{0}\right\rangle .
$$

In addition, we define

$$
\Gamma^{*}(2)=\left\langle\left(\begin{array}{cc}
-\sqrt{2} & -\sqrt{2} \\
1 / \sqrt{2} & 0
\end{array}\right),\left(\begin{array}{cc}
0 & -\sqrt{2} \\
1 / \sqrt{2} & 0
\end{array}\right)\right\rangle .
$$

Since

$$
\left(\begin{array}{cc}
0 & -\sqrt{2} \\
1 / \sqrt{2} & 0
\end{array}\right)^{-1}\left(\begin{array}{cc}
0 & -\sqrt{2} \\
1 / \sqrt{2} & -\sqrt{2}
\end{array}\right)\left(\begin{array}{cc}
0 & -\sqrt{2} \\
1 / \sqrt{2} & 0
\end{array}\right)=\left(\begin{array}{cc}
-\sqrt{2} & -\sqrt{2} \\
1 / \sqrt{2} & 0
\end{array}\right)
$$

we have

$$
\Gamma^{*}(2)=\left\langle\gamma_{1+i}, \gamma_{\sqrt{2} i}\right\rangle
$$




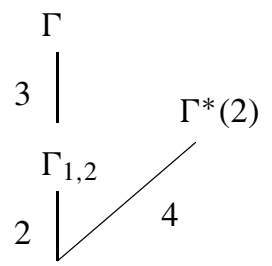

$\Gamma(2)$

FIGURE 2. Inclusion relation.

where

$$
\gamma_{1+i}=\left(\begin{array}{cc}
0 & -\sqrt{2} \\
1 / \sqrt{2} & -\sqrt{2}
\end{array}\right) \quad \text { and } \quad \gamma_{\sqrt{2} i}=\left(\begin{array}{cc}
0 & -\sqrt{2} \\
1 / \sqrt{2} & 0
\end{array}\right)
$$

(see $[\mathbf{N S}]$ ). These groups acts on the upper half complex plane $\mathbb{H}$. The inclusion relation among the above groups is shown in Figure 2.

\subsection{Variants of the lambda function}

Let $\Delta(a, b, c)$ denote the geodesic triangle in the source upper half-plane with vertices $a, b, c \in \overline{\mathbb{H}}=\mathbb{H} \cup \mathbb{R}$. There is a unique biholomorphic map from the triangle $\Delta(a, b, c)$ to the target upper half-plane if we assign the images of the vertices $a, b, c$ say as $\alpha, \beta, \gamma$, respectively. Owing to the Schwarz reflection principle, this map extends to the whole source upper half-space and the target $\mathbb{P}^{1}$; this extended map will be denoted by

$$
\Delta(a, b, c) \longrightarrow(\alpha, \beta, \gamma)
$$

In our cases, for each $\Delta(a, b, c)$, there is a Fuchsian group $\Lambda$ such that the extended map induces an isomorphism of the compactification of the quotient $\mathbb{H} / \Lambda$ and the target $\mathbb{P}^{1}$. For example, the lambda function used in $[\mathbf{K Y}]$ coincides with

$$
\lambda: \Delta(0, \infty,-1) \longrightarrow(0,1, \infty),
$$

which induces the isomorphism $\mathbb{H} / \Gamma(2) \simeq \mathbb{P}^{1}-\{0,1, \infty\}$. We give some variants of the lambda function as in Table 1. 
TABLE 1. Variants of the lambda function.

\begin{tabular}{cccl}
\hline & Name & Group $\Lambda$ & \multicolumn{1}{c}{ Mapping } \\
\hline$(1)$ & $\lambda^{\sharp}$ & $\Gamma(2)$ & $F^{\sharp}(2):=\Delta(0, \infty,-1) \rightarrow(0,1, \infty)$ \\
$(2)$ & $\lambda^{b}$ & $\Gamma(2)$ & $F^{b}(2):=\Delta(\infty, 0,1) \rightarrow(0,1, \infty)$ \\
$(3)$ & $\lambda_{1,2}^{\sharp}$ & $\Gamma_{1,2}$ & $F_{1,2}^{\sharp}:=\Delta(i, \infty,-1) \rightarrow(0,1, \infty)$ \\
$(4)$ & $\lambda_{1,2}^{b}$ & $\Gamma_{1,2}$ & $F_{1,2}^{b}:=\Delta(\infty, i, 1) \rightarrow(0,1, \infty)$ \\
$(5)$ & $J$ & $\Gamma$ & $F:=\Delta\left(\rho_{2}=e^{2 \pi i / 3}, i, \infty\right) \rightarrow(0,1, \infty)$ \\
$(6)$ & $\lambda_{(2)}^{*}$ & $\Gamma^{*}(2)$ & $F^{*}(2):=\Delta(\sqrt{2} i, 1+i, \infty) \rightarrow(0,1, \infty)$ \\
\hline
\end{tabular}

PROPOSITION 2.1. The modular functions above are related as

$$
\begin{gathered}
J=P\left(\lambda^{\sharp}\right)=P\left(\lambda^{b}\right) \quad \text { where } P(\ell)=\frac{4}{27} \frac{\left(1-\ell+\ell^{2}\right)^{3}}{\ell^{2}(1-\ell)^{2}}, \\
\lambda_{1,2}^{\sharp}=P^{\sharp}\left(\lambda^{\sharp}\right)=P^{\sharp}\left(\lambda^{b}\right) \quad \text { where } P^{\sharp}(\ell)=(1-2 \ell)^{2}, \\
\lambda_{1,2}^{b}=P^{b}\left(\lambda^{\sharp}\right)=P^{b}\left(\lambda^{b}\right) \quad \text { where } P^{b}(\ell)=4 \ell(1-\ell), \\
\lambda_{(2)}^{*}=P^{* \sharp}\left(\lambda^{\sharp}\right)=P^{* b}\left(\lambda^{b}\right) \quad \text { where } P^{* \sharp}(\ell)=-\frac{1}{16} \frac{\left(4-4 \ell-\ell^{2}\right)^{2}}{\ell^{2}(1-\ell)} \\
\text { and } P^{* b}(\ell)=-\frac{1}{16} \frac{\left(1-6 \ell+\ell^{2}\right)^{2}}{\ell(1-\ell)^{2}},
\end{gathered}
$$

and

$$
\begin{aligned}
& \lambda^{\sharp}=1-\lambda^{b}, \quad \lambda_{1,2}^{\sharp}=1-\lambda_{1,2}^{b}, \\
& J=P_{1,2}^{\sharp}\left(\lambda_{1,2}^{\#}\right)=P_{1,2}^{b}\left(\lambda_{1,2}^{b}\right) \\
& \text { where } P_{1,2}^{\sharp}(\ell)=\frac{1}{27} \frac{(3+\ell)^{3}}{(1-\ell)^{2}} \text { and } P_{1,2}^{b}=\frac{1}{27} \frac{(4-\ell)^{3}}{\ell^{2}} \text {. }
\end{aligned}
$$

We give some values of the above modular functions in Table 2.

\section{Variants of the kappa function}

In this section we define several variants of the kappa function. 
TABLE 2. Special values.

\begin{tabular}{ccccccc}
\hline & \multicolumn{6}{c}{$z \in \mathbb{H}$} \\
\cline { 2 - 7 } & $\lambda^{\sharp}(z)$ & $\lambda^{\mathrm{b}}(z)$ & $\lambda_{1,2}^{\sharp}(z)$ & $\lambda_{1,2}^{\mathrm{b}}(z)$ & $J(z)$ & $\lambda_{(2)}^{*}(z)$ \\
\hline$i \infty$ & 1 & 0 & 1 & 0 & $\infty$ & $\infty$ \\
$\sqrt{2} i$ & $-2-2 \sqrt{2}$ & $3-2 \sqrt{2}$ & $57-40 \sqrt{2}$ & $-8(7-5 \sqrt{2})$ & $\frac{125}{27}$ & 0 \\
$i$ & $\frac{1}{2}$ & $\frac{1}{2}$ & 0 & 1 & 1 & $-\frac{49}{32}$ \\
0 & 0 & 1 & 1 & 0 & $\infty$ & $\infty$ \\
$\frac{1+i}{2}$ & -1 & 2 & 9 & -8 & 1 & $-\frac{49}{32}$ \\
$\frac{2+\sqrt{2} i}{3}$ & $-2-2 \sqrt{2}$ & $3+2 \sqrt{2}$ & $57+40 \sqrt{2}$ & $-8(7+5 \sqrt{2})$ & $\frac{125}{27}$ & 0 \\
1 & $\infty$ & $\infty$ & $\infty$ & $\infty$ & $\infty$ & $\infty$ \\
$1+i$ & 2 & -1 & 9 & -8 & 1 & 1 \\
$\frac{1+\sqrt{3} i}{2}$ & $\frac{1-\sqrt{3} i}{2}$ & $\frac{1+\sqrt{3} i}{2}$ & -3 & 4 & 0 & $\frac{25}{16}$ \\
\hline
\end{tabular}

\subsection{The function $\kappa_{(2)}^{*}$}

Let us consider the biholomorphic map from $\Delta(\infty, 0,1)$ to $\Delta(\sqrt{2} i, 1+i, \infty)$ that sends $i \infty, 0$ and 1 to $\sqrt{2} i, 1+i$ and $i \infty$, respectively (see Figure 3). By the Schwarz reflection principle, we obtain a mapping $\kappa_{(2)}^{*}: \mathbb{H} \longrightarrow \mathbb{H}$.

Figure 3 and Table 2 tell us that $\lambda^{b}=\lambda_{(2)}^{*} \circ \kappa_{(2)}^{*}$ (see Figure 4). Continuing the functions along a loop around 0 in the target plane, we have

$$
\kappa_{(2)}^{*}(z+2)=-\frac{2}{\kappa_{(2)}^{*}(z)} .
$$

Doing the same at 1 , we have

$$
\kappa_{(2)}^{*}\left(\frac{z}{2 z+1}\right)=-\frac{2}{\kappa_{(2)}^{*}(z)-2} .
$$

We use the symbol $\langle\langle\cdot\rangle\rangle$ to denote the smallest normal subgroup containing - of a relevant group. The correspondences $\gamma_{\infty} \longmapsto \gamma_{\sqrt{2} i}$ and $\gamma_{0} \longmapsto \gamma_{1+i}$ induce an isomorphism $\Gamma(2) /\left\langle\left\langle\gamma_{\infty}{ }^{2}, \gamma_{0}{ }^{4}\right\rangle\right\rangle \cong \Gamma^{*}(2)$. 


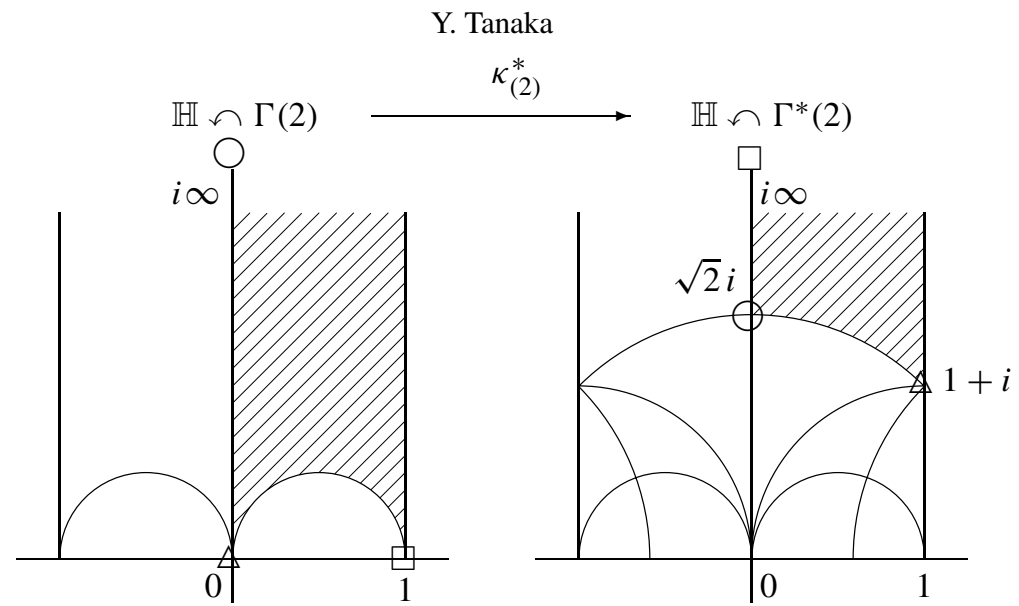

FIGURE 3. The function $\kappa_{(2)}^{*}$.

\subsection{Other kappa functions}

By the same method, we obtain new kappa functions using variants of lambda functions. Starting from the correspondence of the fundamental domains of two modular groups, we obtain variants of kappa functions by the Schwarz reflection principle.

3.2.1. The function $\kappa^{\mathrm{b}}$. This is defined by the equation $\lambda^{\mathrm{b}}(z)=J\left(\kappa^{\mathrm{b}}(z)\right)$ as the extension of the biholomorphic correspondence from $F^{b}(2)$ to $F$ with $(i \infty, 0,1) \rightarrow$ $\left(\rho_{2}, i, i \infty\right)$. The function $\kappa^{\mathrm{b}}$ satisfies

$$
\kappa^{\mathrm{b}}(z+2)=-\frac{1}{\kappa^{\mathrm{b}}(z)} \quad \text { and } \quad \kappa^{\mathrm{b}}\left(\frac{z}{2 z+1}\right)=1-\frac{1}{\kappa^{\mathrm{b}}(z)} .
$$

The correspondences $\gamma_{\infty} \longmapsto \gamma_{\rho_{2}}$ and $\gamma_{0} \longmapsto \gamma_{i}$ induce an isomorphism $\Gamma(2) /\left\langle\left\langle\gamma_{\infty}^{3}, \gamma_{0}^{2}\right\rangle\right\rangle \cong \Gamma$.

3.2.2. The function $\kappa_{1,2}$. This is defined by the equation $\lambda^{b}(z)=\lambda_{1,2}^{\sharp}\left(\kappa_{1,2}(z)\right)$ as the extension of the biholomorphic correspondence from $F^{b}(2)$ to $F_{1,2}^{\sharp}$ with $(i \infty, 0,1) \rightarrow(i, i \infty,-1)$. The function $\kappa_{1,2}$ satisfies

$$
\kappa_{1,2}(z+2)=-\frac{1}{\kappa_{1,2}(z)} \quad \text { and } \quad \kappa_{1,2}\left(\frac{z}{2 z+1}\right)=\frac{\kappa_{1,2}(z)}{2 \kappa_{1,2}(z)+1} .
$$




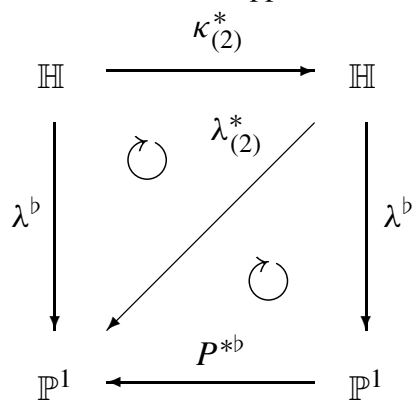

FIGURE 4. Relations among $\kappa_{(2)}^{*}, \lambda$ and $J$.

The correspondences $\gamma_{\infty} \longmapsto \gamma_{i}$ and $\gamma_{0} \longmapsto \gamma_{0}$ induce an isomorphism $\Gamma(2) /\left\langle\left\langle\gamma_{\infty}^{2}\right\rangle\right\rangle \cong \Gamma_{1,2}$.

3.2.3. The function $\check{\kappa}_{1,2}$. This is defined by the equation $\lambda_{1,2}^{b}(z)=J\left(\check{\kappa}_{1,2}(z)\right)$ as the extension of the biholomorphic correspondence from $F_{1,2}^{b}$ to $F$ with $(i \infty, i, 1) \rightarrow$ $\left(\rho_{2}, i, i \infty\right)$. The function $\check{\kappa}_{1,2}$ satisfies

$$
\check{\kappa}_{1,2}(z+2)=-1-\frac{1}{\check{\kappa}_{1,2}(z)} \quad \text { and } \quad \check{\kappa}_{1,2}\left(-\frac{1}{z}\right)=-\frac{1}{\check{\kappa}_{1,2}(z)} .
$$

The correspondences $\gamma_{\infty} \longmapsto \gamma_{\rho_{2}}$ and $\gamma_{i} \longmapsto \gamma_{i}$ induce an isomorphism $\Gamma_{1,2} /\left\langle\left\langle\gamma_{\infty}{ }^{3}\right\rangle\right\rangle \cong \Gamma$.

Relations among these functions are depicted in Figure 5.

\section{Fourier expansion}

Set $q_{n}=e^{\pi i z / n}, \sigma_{k}^{-}(n)=\sum_{d \mid n}(-1)^{k} d^{k}$. Let $R R\left(\left\{b_{n}\right\}\right)$ be the recurrence relations

$$
\begin{aligned}
& R R\left(\left\{b_{n}\right\}\right): 2 n(n-1)(n+1) a_{1} a_{n+1} \\
& =-a_{1}^{2} b_{n}+\sum_{m=1}^{n-1}\left((m+1)(n-m+1)\left(3 n+3 m n-5 m^{2}-4 m\right) a_{m+1} a_{n-m+1}\right. \\
& \left.\quad-b_{n-m} \sum_{j=0}^{m}(j+1)(m-j+1) a_{j+1} a_{m-j+1}\right) \quad(n \geq 2),
\end{aligned}
$$

Each $\kappa$ function in Section 3 has the Fourier series expansion at $z=i \infty$. 


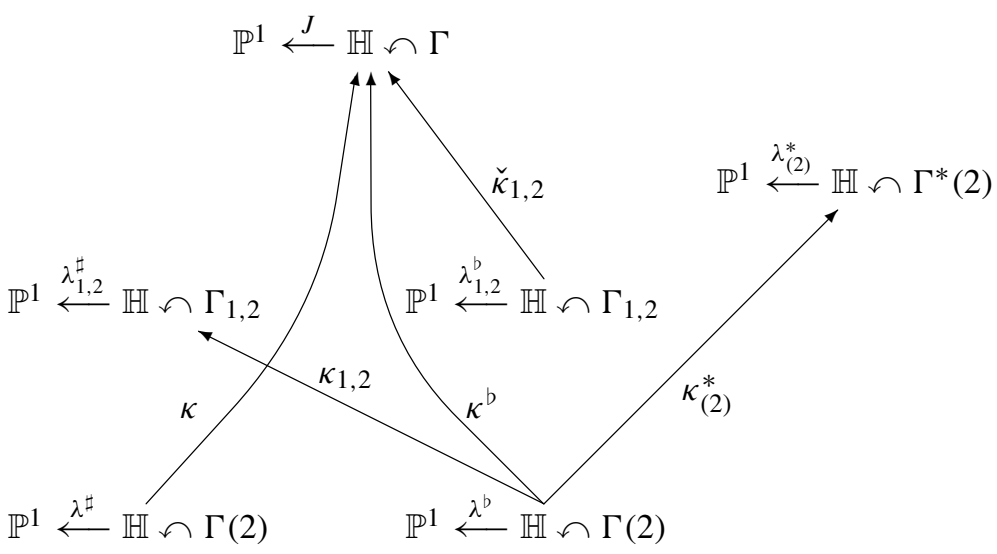

FIGURE 5. Relations among kappa functions.

TABLE 3. $\rho$ and $q$ for each kappa function.

\begin{tabular}{cccccc}
\hline & \multicolumn{5}{c}{$\kappa$} \\
\cline { 2 - 6 } & $\kappa$ & $\kappa^{\mathrm{b}}$ & $\kappa_{1,2}$ & $\check{\kappa}_{1,2}$ & $\kappa_{(2)}^{*}$ \\
\hline$\rho$ & $i$ & $\rho_{2}$ & $i$ & $\rho_{2}$ & $\sqrt{2} i$ \\
$q$ & $q_{2}$ & $q_{3}$ & $q_{2}$ & $q_{3}$ & $q_{2}$ \\
\hline
\end{tabular}

THEOREM 4.1. Our kappa functions have the following Fourier expansions

$$
\kappa(z)=\rho\left(1+\sum_{n=1}^{\infty} a_{n} q^{n}\right),
$$

where $\left\{a_{n}\right\}$ satisfy the recurrence relations $R R\left(\left\{b_{n}\right\}\right) ; \rho, q,\left\{a_{1}, a_{2}, \ldots\right\}, b_{n}, s_{n}, t_{n}$ and $u_{n}$ are given in Tables $3-8$.

Before proving Theorem 4.1, we show several lemmas. Put

$$
=\frac{n}{\pi i} \frac{d}{d z}=q_{n} \frac{d}{d q_{n}} .
$$

LEMMA 4.1. Let

$$
f(z)=\rho \sum_{n=0}^{\infty} a_{n} q 2^{n} \quad\left(a_{0}=1, \rho \in \mathbb{C}\right) .
$$


TABLE 4. Table for $\kappa[\mathbf{K Y}]$.

\begin{tabular}{|c|c|}
\hline$b_{n}$ & $\begin{cases}1 & \text { for } n=0 \\
0 & \text { for } n \text { :odd } \\
(-1)^{n / 2} \frac{64}{9} \sigma_{3}^{-}\left(\frac{n}{2}\right) & \text { for } n \equiv 2 \bmod 4 \\
(-1)^{n / 2} \frac{64}{9} \sigma_{3}^{-}\left(\frac{n}{2}\right)+\frac{80}{9} \sigma_{3}^{-}\left(\frac{n}{4}\right) & \text { for } n \equiv 0 \bmod 4, n \geq 4\end{cases}$ \\
\hline$a_{1}$ & $-i \frac{32}{\sqrt{3}} \frac{\pi^{2}}{\Gamma(1 / 4)^{4}}=-1.0552729262852 \cdots \times i$ \\
\hline \multirow[t]{3}{*}{$a_{2}, a_{3}, \ldots, a_{8}$} & $\left\{\frac{1}{2} a_{1}^{2}, \frac{1}{4} a_{1}^{3}-\frac{16}{27} a_{1}, \frac{1}{8} a_{1}^{4}-\frac{16}{27} a_{1}^{2}, \frac{1}{16} a_{1}^{5}-\frac{4}{9} a_{1}^{3}+\frac{98}{1215} a_{1}\right.$, \\
\hline & $\frac{1}{32} a_{1}{ }^{6}-\frac{8}{27} a_{1}^{4}+\frac{934}{3645} a_{1}^{2}, \frac{1}{64} a_{1}^{7}-\frac{5}{27} a_{1}^{5}+\frac{787}{2430} a_{1}^{3}-\frac{1504}{6561} a_{1}$, \\
\hline & $\left.\frac{1}{128} a_{1}^{8}-\frac{1}{9} a_{1}^{6}+\frac{41}{135} a_{1}^{4}-\frac{9088}{32805} a_{1}^{2}\right\}$ \\
\hline
\end{tabular}

If $f(z)$ satisfies the functional equation $f(z+2)=\rho^{2} / f(z)$, then the recurrent relation among the coefficients $a_{1}, a_{2}, \ldots, a_{n}(n:$ even, $n \geq 2)$ is given by

$$
a_{n}=\sum_{m=1}^{n / 2-1}(-1)^{m-1} a_{m} a_{n-m}+\frac{(-1)^{n / 2-1}}{2} a_{n / 2}{ }^{2} .
$$

In particular, $a_{2}=a_{1}^{2} / 2$.

Proof. Since $q_{2} \mapsto-q_{2}$ as $z \mapsto z+2$, we have

$$
\begin{aligned}
\rho^{2} & =f(z) f(z+2)=\left(\rho \sum_{n=0}^{\infty} a_{n} q_{2}{ }^{n}\right)\left(\rho \sum_{n=0}^{\infty}(-1)^{n} a_{n} q_{2}{ }^{n}\right) \\
& =\rho^{2} \sum_{n=0}^{\infty}\left(\sum_{m=0}^{n}(-1)^{m} a_{m} a_{n-m}\right) q_{2}{ }^{n} .
\end{aligned}
$$

Thus,

$$
\sum_{m=0}^{n}(-1)^{m} a_{m} a_{n-m}=0
$$




\begin{tabular}{|c|c|}
\hline$b_{n}$ & $\begin{cases}1 & \text { for } n=0, \\
0 & \text { for } n \equiv 1,2,4,5 \bmod 6, \\
36(-1)^{n} \sigma_{3}^{-}\left(\frac{n}{3}\right) & \text { for } n \equiv 3 \bmod 6, \\
36(-1)^{n} \sigma_{3}^{-}\left(\frac{n}{3}\right)-20 \sigma_{3}^{-}\left(\frac{n}{6}\right) & \text { for } n \equiv 0 \bmod 6, n \geq 6 .\end{cases}$ \\
\hline$a_{1}$ & $-i \frac{3^{2}}{2^{4 / 3}} \frac{\Gamma(5 / 6)^{2} \Gamma(2 / 3)^{2}}{\pi^{2}}=-0.8454860396348 \cdots \times i$ \\
\hline \multirow[t]{4}{*}{$a_{2}, a_{3}, \ldots, a_{8}$} & $\left\{\frac{1}{\sqrt{3}} e^{\pi i / 6} a_{1}^{2}, \frac{1}{3} e^{\pi i / 3} a_{1}^{3}, \frac{1}{3 \sqrt{3}} e^{\pi i / 2} a_{1}{ }^{4}-\frac{3}{4} a_{1}\right.$, \\
\hline & $\frac{1}{9} e^{2 \pi i / 3} a_{1}{ }^{5}-\frac{\sqrt{3}}{2} e^{\pi i / 6} a_{1}^{2}$, \\
\hline & $\frac{1}{9 \sqrt{3}} e^{5 \pi i} 6 a_{1}{ }^{6}-\frac{3}{4} e^{\pi i / 3} a_{1}{ }^{3}, \frac{1}{27} e^{\pi i} a_{1}{ }^{7}-\frac{1}{\sqrt{3}} e^{\pi i / 2} a_{1}{ }^{4}+\frac{79}{420} a_{1}$, \\
\hline & $\left.\frac{1}{27 \sqrt{3}} e^{7 \pi i / 6} a_{1}{ }^{8}-\frac{5}{12} e^{2 \pi i / 3} a_{1}{ }^{5}+\frac{1577}{1680 \sqrt{3}} e^{\pi i / 6} a_{1}^{2}\right\}$ \\
\hline
\end{tabular}

TABLE 6 . Table for $\kappa_{1,2}$.

\begin{tabular}{|c|c|}
\hline$b_{n}$ & $\begin{array}{ll}1 & \text { for } n=0 \\
0 & \text { for } n \equiv 1,2,3 \bmod 4, \\
16 \sigma_{3}^{-}\left(\frac{n}{4}\right) & \text { for } n \equiv 0 \bmod 4\end{array}$ \\
\hline$a_{1}$ & $-\frac{32 \pi^{2}}{\Gamma(1 / 4)^{4}}=-1.8277863241779 \ldots$ \\
\hline \multirow[t]{2}{*}{$a_{2}, a_{3}, \ldots, a_{8}$} & $\left\{\frac{a_{1}^{2}}{2}, \frac{a_{1}^{3}}{4}, \frac{a_{1}^{4}}{8}, \frac{a_{1}^{5}}{16}+\frac{2}{15} a_{1}, \frac{a_{1}^{6}}{32}+\frac{2}{15} a_{1}^{2}\right.$, \\
\hline & $\left.\frac{a_{1}^{7}}{64}+\frac{a_{1}^{3}}{10}, \frac{a_{1}^{8}}{128}+\frac{a_{1}^{4}}{15}\right\}$ \\
\hline
\end{tabular}


TABLE 7. Table for $\check{\kappa}_{1,2}$.

\begin{aligned} \hline$b_{n} & \begin{cases}1 & \text { for } n=0, \\ 0 & \text { for } n \equiv 1,2 \bmod 3, \\ 16(-1)^{n} \sigma_{3}-\left(\frac{n}{3}\right) & \text { for } n \equiv 0 \bmod 3, n \geq 3 .\end{cases} \\ \left.a_{1}, a_{2}, \ldots, a_{8}\right\} \quad & -i \frac{3^{2}}{2^{2 / 3}} \frac{\Gamma(5 / 6)^{2} \Gamma(2 / 3)^{2}}{\pi^{2}}=-1.3421254287408 \cdots \times i \\ & \frac{1}{\sqrt{3}} e^{\pi i / 6} a_{1}{ }^{2}, \frac{1}{3} e^{\pi i / 3} a_{1}{ }^{3}, \frac{1}{3 \sqrt{3}} e^{\pi i / 2} a_{1}{ }^{4}-\frac{1}{3} a_{1} \\ & , \frac{1}{3 \sqrt{3}} e^{\pi i / 6} a_{1}{ }^{2}\end{aligned}$,

TABLE 8 . Table for $\kappa_{(2)}^{*}$

\begin{tabular}{ll}
\hline$b_{n}$ & $\begin{cases}1 & \text { for } n=0, \\
0 & \text { for } n \equiv 1,3(\bmod 4), \\
12 \sigma_{3}^{-}\left(\frac{n}{2}\right) & \text { for } n \equiv 2(\bmod 4) \\
12 \sigma_{3}^{-}\left(\frac{n}{2}\right)+4 \sigma_{3}^{-}\left(\frac{n}{4}\right) & \text { for } n \equiv 0(\bmod 4), n \geq 4 .\end{cases}$ \\
$a_{1}$ & $-i 2^{\frac{5}{2}} \frac{\Gamma(7 / 8)^{2} \Gamma(5 / 8)^{2}}{\pi^{2}}=-1.4004373562119 \cdots \times i$ \\
$\left\{a_{2}, a_{3}, \ldots, a_{8}\right\}$ & $\left\{\frac{a_{1}{ }^{2}}{2}, \frac{a_{1}{ }^{3}}{4}, \frac{a_{1}{ }^{4}}{8}, \frac{a_{1}{ }^{5}}{16}-\frac{a_{1}}{30}, \frac{a_{1}{ }^{6}}{32}-\frac{a_{1}{ }^{2}}{30}, \frac{a_{1}{ }^{7}}{64}-\frac{a_{1}{ }^{3}}{40}, \frac{a_{1}{ }^{8}}{128}-\frac{a_{1}{ }^{4}}{60}\right\}$ \\
\hline
\end{tabular}


for $n \geq 1$. In the case that $n$ is odd, the left-hand side of this equation is always equal to 0 . In the case that $n$ is even $(n \geq 2)$, we have

$$
\begin{aligned}
& \sum_{m=0}^{n}(-1)^{m} a_{m} a_{n-m} \\
& \quad=2 a_{0} a_{n}+\sum_{m=1}^{n / 2-1}(-1)^{m} a_{m} a_{n-m}+(-1)^{n / 2} a_{n / 2}{ }^{2}+\sum_{m=n / 2-1}^{n-1}(-1)^{m} a_{m} a_{n-m} \\
& \quad=2 a_{n}+2 \sum_{m=1}^{n / 2-1}(-1)^{m} a_{m} a_{n-m}+(-1)^{n / 2} a_{n / 2}{ }^{2}
\end{aligned}
$$

So we obtain Lemma 4.1.

LEMMA 4.2. Let

$$
f(z)=\rho_{2} \sum_{n=0}^{\infty} a_{n} q_{3}^{n} \quad\left(a_{0}=1, \rho_{2}=e^{2 \pi i / 3}\right) .
$$

If $f(z)$ satisfies the functional equation $f(z+2)=-1-1 / f(z)$, then the recurrent relation among the coefficients $a_{1}, a_{2}, \ldots, a_{n}(n \equiv 0,2(\bmod 3), n \geq 2)$ is given by

$$
a_{n}=-\frac{\rho_{2}}{1+\rho_{2}+\rho_{2}{ }^{n+1}} \sum_{m=1}^{n-1} \rho_{2}{ }^{m} a_{m} a_{n-m},
$$

i.e.

$$
a_{n}= \begin{cases}\frac{e^{7 \pi i / 6}}{\sqrt{3}}\left(\sum_{m=1}^{n / 2-1} \delta_{m} a_{m} a_{n-m}+a_{n / 2}{ }^{2}\right) & \text { for } n \equiv 0(\bmod 6), \\ -\frac{i}{\sqrt{3}}\left(\sum_{m=1}^{n / 2-1} \varepsilon_{m} a_{m} a_{n-m}+a_{n / 2}^{2}\right) & \text { for } n \equiv 2(\bmod 6), \\ \frac{e^{7 \pi i / 6}}{\sqrt{3}} \sum_{m=1}^{n / 2-1} \delta_{m} a_{m} a_{n-m} & \text { for } n \equiv 3(\bmod 6), \\ -\frac{i}{\sqrt{3}} \sum_{m=1}^{n / 2-1} \varepsilon_{m} a_{m} a_{n-m} & \text { for } n \equiv 5(\bmod 6),\end{cases}
$$


where

$$
\begin{gathered}
\delta_{m}=\rho_{2}{ }^{k}+\rho_{2}{ }^{-k}= \begin{cases}2 & \text { for } m \equiv 0(\bmod 3), \\
-1 & \text { for } m \equiv 1,2(\bmod 3),\end{cases} \\
\varepsilon_{m}=\rho_{2}{ }^{k}+\rho_{2}{ }^{2-k}= \begin{cases}2 \rho_{2} & \text { for } m \equiv 0,2(\bmod 3), \\
-\rho_{2} & \text { for } m \equiv 1(\bmod 3) .\end{cases}
\end{gathered}
$$

In particular,

$$
a_{2}=\frac{e^{\pi i / 6}}{\sqrt{3}} \frac{a_{1}^{2}}{2} .
$$

Proof. Since $q_{3} \mapsto \rho_{2} q_{3}$ as $z \mapsto z+2$, we have

$$
f(z+2)=\rho \sum_{n=0}^{\infty} \rho_{2}{ }^{n} a_{n} q_{3}{ }^{n} .
$$

By transforming the equation $f(z+2)=-1-1 / f(z)$ to $f(z)(1+f(z+2))=-1$, we have

$$
\begin{aligned}
-1 & =f(z)(1+f(z+2))=\left(\rho_{2} \sum_{n=0}^{\infty} a_{n} q_{3}{ }^{n}\right)\left(1+\rho_{2} \sum_{n=0}^{\infty} \rho_{2}{ }^{n} a_{n} q_{3}{ }^{n}\right) \\
& =\rho_{2} \sum_{n=0}^{\infty} a_{n} q_{3}{ }^{n}+\rho_{2}{ }^{2} \sum_{n=0}^{\infty}\left(\sum_{m=0}^{n}(-1)^{m} a_{m} a_{n-m}\right) q_{2}{ }^{n} \\
& =\rho_{2}+\rho_{2}{ }^{2}+\sum_{n=0}^{\infty}\left(\rho_{2} a_{n}+\rho_{2}{ }^{2} \sum_{m=0}^{n} \rho_{2}{ }^{m} a_{m} a_{n-m}\right) q_{3}{ }^{n} .
\end{aligned}
$$

Thus, we have

$$
\rho_{2} a_{n}+\rho_{2}{ }^{2} \sum_{m=0}^{n} \rho_{2}{ }^{m} a_{m} a_{n-m}=0
$$

i.e.

$$
\left(1+\rho_{2}+\rho_{2}{ }^{n+1}\right) a_{n}=-\rho_{2} \sum_{m=1}^{n-1} \rho_{2}{ }^{m} a_{m} a_{n-m}
$$

for $n \geq 1$. By transforming this equation, we obtain the remaining part of Lemma 4.2.

LEMMA 4.3. Let $q=e^{\pi i z / k}\left(k \in \mathbb{Z}^{+}\right), \rho \in \mathbb{C}$ and

$$
f(z)=\rho \sum_{n=0}^{\infty} a_{n} q^{n} \quad\left(a_{0}=1\right) .
$$


Suppose

$$
2\{f ; z\}=-\left(\frac{k}{\pi i}\right)^{2} \sum_{n=0}^{\infty} b_{n} q^{n} \quad\left(b_{0}=1\right),
$$

where $\{f ; z\}$ is Schwarzian derivative. Then the recurrent relation among the coefficients $a_{1}, a_{2}, \ldots, a_{n+1}$ is given by

$$
\begin{aligned}
& 2 n(n-1)(n+1) a_{1} a_{n+1} \\
& =-a_{1}{ }^{2} b_{n}+\sum_{m=1}^{n-1}\left((m+1)(n-m+1)\left(3 n+3 m n-5 m^{2}-4 m\right) a_{m+1} a_{n-m+1}\right. \\
& \left.\quad-b_{n-m} \sum_{l=0}^{m}(l+1)(m-l+1) a_{l+1} a_{m-l+1}\right) .
\end{aligned}
$$

Proof. By the definition of Schwarzian derivative we have

$$
2\{f ; z\}=\left(\frac{k}{\pi i}\right)^{2} \frac{2 f(z)^{\prime} f(z)^{\prime \prime \prime}-3\left(f(z)^{\prime \prime}\right)^{2}}{\left(f(z)^{\prime}\right)^{2}}=-\left(\frac{k}{\pi i}\right)^{2} \sum_{n=0}^{\infty} b_{n} q^{n} .
$$

Hence, we have

$$
2 f(z)^{\prime} f(z)^{\prime \prime \prime}-3\left(f(z)^{\prime \prime}\right)^{2}=\left(-\sum_{n=0}^{\infty} b_{n} q^{n}\right)\left(f(z)^{\prime}\right)^{2}
$$

Since $f(z)^{\prime}=\rho q \sum_{n=0}^{\infty}(n+1) a_{n+1} q^{n}, f(z)^{\prime \prime}=\rho q \sum_{n=0}^{\infty}(n+1)^{2} a_{n+1} q^{n}, f(z)^{\prime \prime \prime}=$ $\rho q \sum_{n=0}^{\infty}(n+1)^{3} a_{n+1} q^{n}$, we have the Fourier expansions of both sides of (4.1) as follows:

(i) left-hand side of (4.1)

$$
\begin{aligned}
2 f(z)^{\prime} f(z)^{\prime \prime \prime}-3\left(f(z)^{\prime \prime}\right)^{2} & \\
= & 2\left(\rho q \sum_{n=0}^{\infty}(n+1) a_{n+1} q^{n}\right)\left(\rho q \sum_{n=0}^{\infty}(n+1)^{3} a_{n+1} q^{n}\right) \\
& -3\left(\rho q \sum_{n=0}^{\infty}(n+1)^{2} a_{n+1} q^{n}\right)^{2} \\
= & -\rho^{2} q^{2} \sum_{n=0}^{\infty}\left(\sum_{m=0}^{n}(m+1)^{2}(n-m+1)(3 n-5 m+1) a_{m+1} a_{n-m+1}\right) q^{n}
\end{aligned}
$$


(ii) right-hand side of (4.1)

$$
\begin{aligned}
& \left(-\sum_{n=0}^{\infty} b_{n} q^{n}\right)\left(f(z)^{\prime}\right)^{2} \\
& \quad=-\left(\sum_{n=0}^{\infty} b_{n} q^{n}\right)\left(\rho q \sum_{n=0}^{\infty}(n+1) a_{n+1} q^{n}\right) \\
& \quad=-\rho^{2} q^{2} \sum_{n=0}^{\infty}\left(\sum_{m=0}^{n}\left(b_{n-m} \sum_{l=0}^{m}(l+1)(m-l+1) a_{l+1} a_{m-l+1}\right)\right) q^{n} .
\end{aligned}
$$

By equating the coefficients of $q^{n}$ of the above two Fourier expansions we have

$\sum_{m=0}^{n}(m+1)^{2}(n-m+1) a_{m+1} a_{n-m+1}=\sum_{m=0}^{n}\left(b_{n-m} \sum_{l=0}^{m}(l+1)(m-l+1) a_{l+1} a_{m-l+1}\right)$,

which induces $a_{1}, a_{2}, \ldots, a_{n}$ and $a_{n+1}$; solving $a_{1}, a_{2}, \ldots, a_{n}$ and $a_{n+1}$ from this equation we obtain Lemma 4.3 .

Now we recall the Eisenstein series

$$
\begin{gathered}
E_{2}(z)=1-24 \sum_{n=1}^{\infty} \sigma_{1}(n) e^{2 \pi i n z}, \quad E_{4}(z)=1+240 \sum_{n=1}^{\infty} \sigma_{3}(n) e^{2 \pi i n z}, \\
E_{6}(z)=1-504 \sum_{n=1}^{\infty} \sigma_{5}(n) e^{2 \pi i n z} .
\end{gathered}
$$

LEMMA 4.4. We have the following limit values:

$$
\begin{gathered}
\lim _{z \rightarrow i \infty} \frac{1}{q_{2}} \frac{d \lambda^{\sharp}(z)}{d q_{2}}=-32, \quad \lim _{z \rightarrow i \infty} \frac{1}{q_{2}} \frac{d \lambda^{b}(z)}{d q_{2}}=32, \\
\lim _{z \rightarrow i \infty} \frac{1}{q_{2}} \frac{d}{d q_{2}}\left(q_{2} \frac{d \lambda^{b}(z)}{d q_{2}}\right)=64, \quad \lim _{z \rightarrow i \infty} \frac{1}{q_{2}} \frac{d \lambda_{1,2}^{\#}(z)}{d q_{2}}=-256, \\
\lim _{z \rightarrow i \infty} \frac{1}{q_{2}} \frac{d \lambda_{1,2}^{\sharp}(\tau)}{d \tau}=-\frac{2}{3} \pi^{2} i a_{1} E_{4}(i) \quad \text { for } \tau=\kappa_{1,2}(z), \\
\lim _{z \rightarrow i \infty} \frac{1}{q_{2}} \frac{d J(\tau)}{d \tau}=-2 \pi^{2} i a_{1} E_{4}(i) \quad \text { for } \tau=i\left(1+a_{1} q_{2}+a_{2} q_{2}^{2}+a_{3} q_{2}^{3}+\cdots\right), \\
\lim _{z \rightarrow i \infty} \frac{1}{q_{2}} \frac{d \lambda_{(2)}^{*}(\tau)}{d \tau}=\frac{2^{5 / 2}}{(\sqrt{2}-1)^{2}} \pi^{2} i a_{1} \theta_{2}(\sqrt{2} i)^{8} \quad \text { for } \tau=\kappa_{(2)}^{*}(z), \\
\lim _{z \rightarrow i \infty} \frac{d^{2} \lambda_{(2)}^{*}(\tau)}{d \tau^{2}}=\frac{2^{2}}{(\sqrt{2}-1)^{2}} \pi^{2} \theta_{2}(\sqrt{2} i)^{8} \quad \text { for } \tau=\kappa_{(2)}^{*}(z) .
\end{gathered}
$$


Proof. Since

$$
\begin{gathered}
\frac{d \lambda^{\sharp}(z)}{d q_{2}}=\frac{d}{d q_{2}}\left(1-16 q_{2}{ }^{2}+128 q_{2}{ }^{4}-704 q_{2}{ }^{6}+\cdots\right) \\
=-32 q_{2}+512 q_{2}{ }^{3}-4224 q_{2}{ }^{5}+\cdots \\
\frac{d}{d q_{2}}\left(q_{2} \frac{d \lambda^{b}(z)}{d q_{2}}\right)=-64 q_{2}+2048 q_{2}{ }^{3}-25344 q_{2}{ }^{5}+\cdots
\end{gathered}
$$

and $\lambda^{\sharp}(z)=1-\lambda^{b}(z)$, we obtain

$$
\begin{gathered}
\lim _{z \rightarrow i \infty} \frac{1}{q_{2}} \frac{d \lambda^{\sharp}(z)}{d q_{2}}=-32, \\
\lim _{z \rightarrow i \infty} \frac{1}{q_{2}} \frac{d \lambda^{b}(z)}{d q_{2}}=32 \text { and } \lim _{z \rightarrow i \infty} \frac{1}{q_{2}} \frac{d}{d q_{2}}\left(q_{2} \frac{d \lambda^{b}(z)}{d q_{2}}\right)=64 .
\end{gathered}
$$

Let $\tau=\kappa_{1,2}(z)$. Since $J(\tau)=P_{1,2}^{\sharp}\left(\lambda_{1,2}^{\sharp}(\tau)\right)$, we have

$$
-2 \pi i \frac{E_{6}(\tau)}{E_{4}(\tau)} J(\tau)=\frac{d J(\tau)}{d \tau}=\frac{1}{27} \frac{\left(\lambda_{1,2}^{\sharp}(\tau)-9\right)\left(\lambda_{1,2}^{\sharp}(\tau)+3\right)^{2}}{\left(\lambda_{1,2}^{\sharp}(\tau)-1\right)^{2}} .
$$

We also have $\lambda_{1,2}^{\sharp}\left(\kappa_{1,2}(z)\right)=\lambda^{b}(z)$ and $\lambda^{b}(i \infty)=0$. So we obtain

$$
\begin{aligned}
\lim _{z \rightarrow i \infty} \frac{1}{q_{2}} \frac{d \lambda_{1,2}^{\sharp}(\tau)}{d \tau} & =\lim _{z \rightarrow i \infty} \frac{1}{q_{2}}\left(\frac{E_{6}\left(\kappa_{1,2}(z)\right)}{E_{4}\left(\kappa_{1,2}(z)\right)} \frac{\left(\lambda^{b}(z)+3\right)\left(\lambda^{b}(z)-1\right)}{\lambda^{b}(z)-9}\right) \\
& =-\frac{2}{3} \pi^{2} i a_{1} E_{4}(i) .
\end{aligned}
$$

Let $\tau=i\left(1+a_{1} q_{2}+a_{2} q_{2}^{2}+a_{3} q_{2}^{3}+\cdots\right)$. Since

$$
\begin{aligned}
\frac{d J(\tau)}{d \tau}= & -2 \pi i \frac{E_{6}(\tau)}{E_{4}(\tau)} J(\tau)=-2 \pi i \frac{E_{4}(\tau)^{2} E_{6}(\tau)}{E_{4}(\tau)^{3}-E_{6}(\tau)^{2}} \\
& \text { and } \lim _{z \rightarrow i \infty} \frac{E_{6}(\tau)}{q_{2}}=\pi a_{1} E_{4}(i)^{2},
\end{aligned}
$$

we obtain

$$
\lim _{z \rightarrow i \infty} \frac{1}{q_{2}} \frac{d J(\tau)}{d \tau}=-2 \pi i \lim _{z \rightarrow i \infty} \frac{E_{6}(\tau)}{q_{2}} \frac{1}{E_{4}(\tau)} J(\tau)=-2 \pi^{2} i a_{1} E_{4}(i) .
$$

Let $\tau=\kappa_{(2)}^{*}(z), N(z)=1-6 \lambda_{(2)}^{b}(z)+\lambda_{(2)}^{b}(z)^{2}$ and $D(z)=16 \lambda^{b}(z)\left(1-\lambda^{b}(z)\right)$. Then we have

$$
\lambda_{(2)}^{*}(z)=P^{*}\left(\lambda^{b}(z)\right)=-\frac{N(z)^{2}}{D(z)} \text {. }
$$


Since $\lambda^{b}(z)^{\prime}=2 \theta_{2}(z)^{8}\left(1-\lambda^{b}(z)\right)$ and $\lambda^{b}(\sqrt{2} i)=3-2 \sqrt{2}$, we have

$$
\lim _{z \rightarrow i \infty} \frac{d \lambda^{b}(\tau)}{\tau}=\lim _{z \rightarrow i \infty} \frac{\pi i}{2} \lambda^{b}(\tau)^{\prime}=2(\sqrt{2}-1) \pi i \theta_{2}(\sqrt{2} i)^{4} .
$$

Hence,

$$
\begin{gathered}
\lim _{z \rightarrow i \infty} \frac{d N(\tau)}{d \tau}=\lim _{z \rightarrow i \infty}\left(-6+2 \lambda^{b}(\tau)\right) \frac{d \lambda^{b}(\tau)}{d \tau}=-2^{7 / 2}(\sqrt{2}-1) \pi i \theta_{2}(\sqrt{2} i)^{4}, \\
\lim _{z \rightarrow i \infty} \frac{1}{q_{2}} N(\tau)=\lim _{z \rightarrow i \infty} \frac{d N(\tau) / d z}{(\pi i / 2) q_{2}}=\lim _{z \rightarrow i \infty} \frac{(\pi i / 2) \tau^{\prime}(d N(\tau) / d \tau)}{(\pi i / 2) q_{2}} \\
=2^{4}(\sqrt{2}-1) \pi a_{1} \theta_{2}(\sqrt{2} i)^{4} .
\end{gathered}
$$

So we obtain the following two limit values, noting that $N(\sqrt{2} i)=0$ :

$$
\begin{aligned}
\lim _{z \rightarrow i \infty} \frac{1}{q_{2}} \frac{d \lambda_{(2)}^{*}(\tau)}{d \tau} & =\lim _{z \rightarrow i \infty} \frac{1}{q_{2}} \frac{d}{d \tau}\left(N(\tau)^{2} \frac{1}{D(\tau)}\right) \\
& =\lim _{z \rightarrow i \infty}\left(-\frac{1}{q_{2}} 2 N(\tau) \frac{d N(\tau)}{d \tau} \frac{1}{D(\tau)}+\frac{1}{q_{2}} N(\tau)^{2} \frac{1}{D(\tau)^{2}} \frac{d D(\tau)}{d \tau}\right) \\
& =2^{5 / 2}(\sqrt{2}-1)^{-2} \pi^{2} i a_{1} \theta_{2}(\sqrt{2} i)^{8}
\end{aligned}
$$

and

$$
\begin{aligned}
\lim _{z \rightarrow i \infty} \frac{d^{2} \lambda_{(2)}^{*}(\tau)}{d \tau^{2}}= & \lim _{z \rightarrow i \infty}\left(N ( \tau ) \left(-\frac{d^{2} N(\tau)}{d \tau^{2}} \frac{1}{D(\tau)}+2 \frac{d N(\tau)}{d \tau} \frac{1}{D(\tau)^{2}} \frac{d D(\tau)}{d \tau}\right.\right. \\
& \left.\left.+N(\tau) \frac{d}{d \tau}\left(\frac{1}{D(\tau)^{2}} \frac{d D(\tau)}{d \tau}\right)\right)-2 \frac{1}{D(\tau)}\left(\frac{d N(\tau)}{d \tau}\right)^{2}\right) \\
= & 2^{2}(\sqrt{2}-1)^{-2} \pi^{2} \theta_{2}(\sqrt{2} i)^{8} .
\end{aligned}
$$

LEMMA 4.5. We have the following limit values:

$$
\begin{gathered}
\lim _{z \rightarrow i \infty} \frac{1}{q_{3}^{2}} \frac{d \lambda^{b}(z)}{d q_{3}}=48, \quad \lim _{z \rightarrow i \infty} \frac{1}{q_{3}^{2}} \frac{d \lambda_{1,2}^{b}(z)}{d q_{3}}=192, \\
\lim _{z \rightarrow i \infty} \frac{1}{q_{3}^{2}} \frac{d J(\tau)}{d \tau} \\
=-\frac{8}{9} \pi^{3} i \rho_{3}{ }^{2} a_{1}{ }^{2} E_{6}\left(\rho_{2}\right) \quad \text { for } \tau=\rho_{2}\left(1+a_{1} q_{3}+a_{2} q_{3}{ }^{2}+a_{3} q_{3}{ }^{3}+\cdots\right) .
\end{gathered}
$$

Proof. Since

$$
\begin{gathered}
\lambda^{b}(z)=16 q_{3}{ }^{3}-128 q_{3}{ }^{6}+704 q_{3}{ }^{9}-\cdots, \\
\lambda_{1,2}^{b}(z)=P^{b}\left(\lambda^{b}(z)\right)=64 q_{3}{ }^{3}-1536 q_{3}{ }^{6}+19200 q_{3}{ }^{12}+\cdots,
\end{gathered}
$$


we obtain

$$
\lim _{z \rightarrow i \infty} \frac{1}{q_{3}^{2}} \frac{d \lambda^{b}(z)}{d q_{3}}=48 \text { and } \lim _{z \rightarrow i \infty} \frac{1}{q_{3}^{2}} \frac{\lambda_{1,2}^{b}(z)}{d q_{3}}=192 .
$$

Let $\tau=\rho_{2}\left(1+a_{1} q_{3}+a_{2} q_{3}^{2}+a_{3} q_{3}^{3}+\ldots\right)$. Since

$$
\frac{d J(\tau)}{d \tau}=-2 \pi i \frac{E_{6}(\tau)}{E_{4}(\tau)} J(\tau)=-2 \pi i \frac{E_{4}(\tau)^{2} E_{6}(\tau)}{E_{4}(\tau)^{3}-E_{6}(\tau)^{2}}
$$

and

$$
\lim _{z \rightarrow i \infty} \frac{E_{4}(\tau)}{q_{3}}=-\frac{2}{3} \pi i \rho_{3} a_{1} E_{6}\left(\rho_{2}\right)
$$

we obtain

$$
\begin{aligned}
\lim _{z \rightarrow i \infty} \frac{1}{q_{3}^{2}} \frac{d J(\tau)}{d \tau} & =-2 \pi i \lim _{z \rightarrow i \infty}\left(\frac{E_{4}(\tau)}{q_{3}}\right)^{2} \frac{E_{6}(\tau)}{E_{4}(\tau)^{3}-E_{6}(\tau)^{2}} \\
& =-\frac{8}{9} \pi^{3} i \rho_{3}{ }^{2} a_{1}^{2} E_{6}\left(\rho_{2}\right)
\end{aligned}
$$

Now we prove Theorem 4.1 for $\kappa^{b}$ and $\kappa_{(2)}^{*}$. The others can be proved in a similar way.

Proof of Theorem 4.1. We first prove Theorem 4.1 for $\kappa^{b}$.

Since

$$
\kappa^{b}(z+2)=-1-\frac{1}{\kappa^{b}(z)}, \quad \kappa^{b}(z+6)=\kappa^{b}(z),
$$

and $\kappa^{b}(i \infty)=\rho_{2}, \kappa^{b}(z)$ admits the Fourier expansion

$$
\rho_{2}\left(1+a_{1} q_{3}+a_{2} q_{3}^{2}+a_{3} q_{3}^{3}+\cdots\right) .
$$

Let $x=\lambda^{b}(z)$. By the properties of the Schwarzian derivative, we have

$$
\begin{gathered}
2\left\{\left(\lambda^{b}\right)^{-1} ; x\right\}=\frac{1}{x^{2}}+\frac{1}{(1-x)^{2}}+\frac{1}{x(1-x)}, \\
2\left\{J^{-1} ; x\right\}=\frac{1-(1 / 3)^{2}}{x^{2}}+\frac{1-(1 / 2)^{2}}{(1-x)^{2}}+\frac{1-(1 / 3)^{2}-(1 / 2)^{2}}{x(1-x)}
\end{gathered}
$$

and

$$
\begin{aligned}
2\left\{\kappa^{b} ; z\right\} & =2\left\{\lambda^{b} ; z\right\}-2\left\{J^{-1} ; x\right\}\left(\frac{d x}{d z}\right)^{2} \\
& =\left(-\left(2\left\{\left(\lambda^{b}\right)^{-1} ; x\right\}\right)+\left(2\left\{J^{-1} ; x\right\}\right)\right)\left(\frac{d x}{d z}\right)^{2}=-\frac{1}{36} \frac{4+5 x}{x^{2}(1-x)^{2}}\left(\frac{d x}{d z}\right)^{2}
\end{aligned}
$$


Multiplying both sides by $(3 / \pi i)^{2}$, we have

$$
\begin{aligned}
\frac{2 \kappa^{\mathrm{b}}(z)^{\prime} \kappa^{\mathrm{b}}(z)^{\prime \prime \prime}-3\left(\kappa^{\mathrm{b}}(z)^{\prime \prime}\right)}{\left(\kappa^{\mathrm{b}}(z)^{\prime}\right)^{2}} & =-\frac{1}{36} \frac{4+5 \lambda^{\mathrm{b}}(z)}{\lambda^{\mathrm{b}}(z)^{2}\left(1-\lambda^{\mathrm{b}}(z)\right)^{2}}\left(\lambda^{\mathrm{b}}(z)^{\prime}\right)^{2} \\
& =-\frac{1}{4}\left(9 \theta_{3}(z)^{8}-5 \theta_{0}(z)^{4} \theta_{3}(z)^{4}\right) .
\end{aligned}
$$

(Note that $\lambda^{b}(z)^{\prime}=3 \theta_{2}(z)^{4}\left(1-\lambda^{b}(z)\right)$ for $q_{3}$.) Put

$$
\frac{1}{4}\left(9 \theta_{3}(z)^{8}-5 \theta_{0}(z)^{4} \theta_{3}(z)^{4}\right)=\sum_{n=0}^{\infty} b_{n} q_{3}{ }^{n} .
$$

By the formulas

$$
\theta_{0}(z)^{4} \theta_{3}(z)^{4}=1+16 \sum_{n=1}^{\infty} \sigma_{3}^{-}(n) q_{3}{ }^{6 n} \text { and } \theta_{3}(z)^{8}=1+16 \sum_{n=1}^{\infty} \sigma_{3}^{-}(n) q_{3}{ }^{3 n},
$$

we have

$$
b_{n}= \begin{cases}1 & \text { for } n=0, \\ 0 & \text { for } n \equiv 1,2,4,5 \bmod 6, \\ 36(-1)^{n} \sigma_{3}^{-}\left(\frac{n}{3}\right) & \text { for } n \equiv 3 \bmod 6, \\ 36(-1)^{n} \sigma_{3}^{-}\left(\frac{n}{3}\right)-20 \sigma_{3}^{-}\left(\frac{n}{6}\right) & \text { for } n \equiv 0 \bmod 6 .\end{cases}
$$

By Lemmas 4.2 and 4.3, we obtain the recurrent relation of $\left\{a_{n}\right\}$ in Theorem 4.1. Now we calculate $a_{1}$. Differentiating the identity $J\left(\kappa^{b}(z)\right)=\lambda^{b}(z)$ and multiplying both sides by $3 / \pi i$, we have

$$
\kappa^{b}(z)^{\prime} \frac{d J(\tau)}{d \tau}=\lambda^{b}(z)^{\prime}
$$

where $\tau=\kappa^{b}(z)$. Multiplying both sides by $1 / q_{3}^{3}$ and using Lemma 4.5 , we have

$$
\rho_{2} a_{1} \times\left(-\frac{8}{9} \pi^{3} i \rho_{3}^{2} a_{1}^{2} E_{6}\left(\rho_{2}\right)\right)=48
$$

as $z \rightarrow i \infty$. By Proposition A.2 and (4.2), we obtain Table 5 in Theorem 4.1.

We now prove Theorem 4.1 for $\kappa_{(2)}^{*}$. Since

$$
\kappa_{(2)}^{*}(z+2)=-\frac{2}{\kappa_{(2)}^{*}(z)}, \quad \kappa_{(2)}^{*}(z+4)=\kappa_{(2)}^{*}(z),
$$

and $\kappa_{(2)}^{*}(i \infty)=\sqrt{2} i, \kappa_{(2)}^{*}(z)$ admits the Fourier expansion

$$
\sqrt{2} i\left(1+a_{1} q_{2}+a_{2} q_{2}^{2}+a_{3} q_{2}^{3}+\cdots\right) \text {. }
$$


Let $x=\lambda^{b}(z)$. By the properties of the Schwarzian derivative, we have

$$
\begin{gathered}
2\left\{\left(\lambda^{b}\right)^{-1} ; x\right\}=\frac{1}{x^{2}}+\frac{1}{(1-x)^{2}}+\frac{1}{x(1-x)}, \\
2\left\{\left(\lambda_{(2)}^{*}\right)^{-1} ; x\right\}=\frac{1-(1 / 2)^{2}}{x^{2}}+\frac{1-(1 / 4)^{2}}{(1-x)^{2}}+\frac{1-(1 / 2)^{2}-(1 / 4)^{2}}{x(1-x)}
\end{gathered}
$$

and

$$
\begin{aligned}
2\left\{\kappa_{(2)}^{*} ; z\right\} & =2\left\{\lambda^{b} ; z\right\}-2\left\{\left(\lambda_{(2)}^{*}\right)^{-1} ; x\right\}\left(\frac{d x}{d z}\right)^{2} \\
& =\left(-\left(2\left\{\left(\lambda^{b}\right)^{-1} ; x\right\}\right)+\left(2\left\{\left(\lambda_{(2)}^{*}\right)^{-1} ; x\right\}\right)\right)\left(\frac{d x}{d z}\right)^{2} \\
& =-\frac{1}{16} \frac{4-3 x}{x^{2}(1-x)^{2}}\left(\frac{d x}{d z}\right)^{2} .
\end{aligned}
$$

Multiplying both sides by $(2 / \pi i)^{2}$, we have

$$
\begin{aligned}
\frac{2 \kappa_{(2)}^{*}(z)^{\prime} \kappa_{(2)}^{*}(z)^{\prime \prime \prime}-3\left(\kappa_{(2)}^{*}(z)^{\prime \prime}\right)^{2}}{\left(\kappa_{(2)}^{*}(z)^{\prime}\right)^{2}} & =-\frac{1}{16} \frac{4-3 \lambda^{b}(z)}{\lambda^{b}(z)^{2}\left(1-\lambda^{b}(z)\right)^{2}}\left(\lambda^{b}(z)^{\prime}\right)^{2} \\
& =-\frac{1}{4}\left(3 \theta_{0}(z)^{4} \theta_{3}(z)^{4}+\theta_{3}(z)^{8}\right) .
\end{aligned}
$$

(Note that $\lambda^{b}(z)^{\prime}=2 \theta_{2}(z)^{4}\left(1-\lambda^{b}(z)\right)$ for $q_{2}$.) Put

$$
\frac{1}{4}\left(3 \theta_{0}(z)^{4} \theta_{3}(z)^{4}+\theta_{3}(z)^{8}\right)=\sum_{n=0}^{\infty} b_{n} q 2^{n} .
$$

By the formula

$$
\theta_{0}(z)^{4} \theta_{3}(z)^{4}=1+16 \sum_{n=1}^{\infty} \sigma_{3}^{-}(n) q_{2}{ }^{4 n} \quad \text { and } \quad \theta_{3}(z)^{8}=1+16 \sum_{n=1}^{\infty} \sigma_{3}^{-}(n) q_{2}{ }^{2 n},
$$

we have

$$
b_{n}= \begin{cases}1 & \text { for } n=0, \\ 0 & \text { for } n \equiv 1,3(\bmod 4), \\ 12 \sigma_{3}^{-}\left(\frac{n}{2}\right) & \text { for } n \equiv 2(\bmod 4), \\ 12 \sigma_{3}^{-}\left(\frac{n}{2}\right)+4 \sigma_{3}^{-}\left(\frac{n}{4}\right) & \text { for } n \equiv 0(\bmod 4),\end{cases}
$$

By Lemmas 4.1 and 4.3, we obtain the recurrent relation of $\left\{a_{n}\right\}$ in Theorem 4.1. 
Now we calculate $a_{1}$. Differentiating the identity $\lambda_{(2)}^{*}\left(\kappa_{(2)}^{*}(z)\right)=\lambda^{b}(z)$ twice and multiplying both sides by $(2 / \pi i)^{2}$, we have

$$
\kappa_{(2)}^{*}(z)^{\prime \prime} \frac{d \lambda_{(2)}^{*}(\tau)}{d \tau}+\left(\kappa_{(2)}^{*}(z)^{\prime}\right)^{2} \frac{d^{2} \lambda_{(2)}^{*}(\tau)}{d \tau^{2}}=\lambda^{b}(z)^{\prime \prime}
$$

where $\tau=\kappa_{(2)}^{*}(z)$. Multiplying both sides by $1 / q_{2}^{2}$ and using Lemma 4.4, we have

$$
\sqrt{2} i a_{1} \times \frac{2^{5 / 2}}{(\sqrt{2}-1)^{2}} \pi^{2} i a_{1} \theta_{2}(\sqrt{2} i)^{8}+\left(\sqrt{2} i a_{1}\right)^{2} \times \frac{2^{2}}{(\sqrt{2}-1)^{2}} \pi^{2} \theta_{2}(\sqrt{2} i)^{8}=64
$$

as $z \rightarrow i \infty$. Using Proposition A.1 and (4.3) we obtain Table 8 in Theorem 4.1.

\section{Appendix A}

In this appendix we give some special values of the elliptic theta functions $\theta_{0}, \theta_{2}$ and $\theta_{3}$, and the Eisenstein series $E_{4}$ and $E_{6}$ used in this paper.

\section{A.1. Elliptic theta function}

Proposition A.1. Values at $z=i, e^{\pi i / 3}, e^{2 \pi i / 3}$ and $\sqrt{2} i$ of $\theta_{0}(z)^{4}, \theta_{2}(z)^{4}$ and $\theta_{3}(z)^{4}$ are given in Table A.1.

Proof. Let $k=k(z)=\theta_{2}(z)^{2} / \theta_{3}(z)^{2}$. Since

$$
K(k)=\int_{0}^{1} \frac{d x}{\sqrt{\left(1-x^{2}\right)\left(1-k^{2} x^{2}\right)}}=\frac{\pi}{2} \theta_{3}(z)^{2}=\frac{\pi}{2}{ }_{2} F_{1}\left(\frac{1}{2}, \frac{1}{2} ; 1 ; k^{2}\right),
$$

we have the identity

$$
\theta_{3}(z)^{2}={ }_{2} F_{1}\left(\frac{1}{2}, \frac{1}{2} ; 1 ; \lambda^{b}(z)\right) .
$$

At $z=i$, by using the inversion formula of gamma function and the equation

$$
{ }_{2} F_{1}\left(2 a, 1-2 a ; 2 c ; \frac{1}{2}\right)=\frac{2^{1-2 c} \Gamma(2 c) \Gamma(1 / 2)}{\Gamma(a+c) \Gamma(c-a+1 / 2)}
$$

(see [EMOT, §2.1.5, p. 68]) with $a=\frac{1}{4}$ and $c=\frac{1}{2}$, we have

$$
\theta_{3}(i)^{4}={ }_{2} F_{1}\left(\frac{1}{2}, \frac{1}{2} ; 1 ; \lambda^{b}(i)\right)^{2}={ }_{2} F_{1}\left(\frac{1}{2}, \frac{1}{2} ; 1 ; \frac{1}{2}\right)^{2}=\frac{\Gamma(1 / 4)^{4}}{2^{2} \pi^{3}} .
$$

Since

$$
\frac{\theta_{2}(i)^{4}}{\theta_{3}(i)^{4}}=\lambda^{b}(i)=\frac{1}{2} \quad \text { and } \quad \frac{\theta_{0}(i)^{4}}{\theta_{3}(i)^{4}}=1-\lambda^{b}(i)=\frac{1}{2},
$$


TABLE A.1. Special values of $\theta_{0}, \theta_{2}$ and $\theta_{3}$.

\begin{tabular}{ccccc}
\hline & $z=i$ & $z=\rho_{1}=e^{\pi i / 3}$ & $z=\rho_{2}=e^{2 \pi i / 3}$ & $z=\sqrt{2} i$ \\
\hline$\theta_{0}(z)^{4}$ & $\frac{\Gamma(1 / 4)^{4}}{2^{3} \pi^{3}}$ & $\frac{2^{2} \pi e^{11 \pi i / 6}}{3^{3 / 2} \Gamma(2 / 3)^{2} \Gamma(5 / 6)^{2}}$ & $\frac{2^{2} \pi e^{\pi i / 6}}{3^{3 / 2} \Gamma(2 / 3)^{2} \Gamma(5 / 6)^{2}}$ & $\frac{\pi}{2^{1 / 2} \Gamma(5 / 8)^{2} \Gamma(7 / 8)^{2}}$ \\
$\theta_{2}(z)^{4}$ & $\frac{\Gamma(1 / 4)^{4}}{2^{3} \pi^{3}}$ & $\frac{2^{2} \pi e^{\pi i / 2}}{3^{3 / 2} \Gamma(2 / 3)^{2} \Gamma(5 / 6)^{2}}$ & $\frac{2^{2} \pi e^{-\pi i / 2}}{3^{3 / 2} \Gamma(2 / 3)^{2} \Gamma(5 / 6)^{2}}$ & $\frac{(\sqrt{2}-1) \pi}{2^{3 / 2} \Gamma(5 / 8)^{2} \Gamma(7 / 8)^{2}}$ \\
$\theta_{3}(z)^{4}$ & $\frac{\Gamma(1 / 4)^{4}}{2^{2} \pi^{3}}$ & $\frac{2^{2} \pi e^{\pi i / 6}}{3^{3 / 2} \Gamma(2 / 3)^{2} \Gamma(5 / 6)^{2}}$ & $\frac{2^{2} \pi e^{11 \pi i / 6}}{3^{3 / 2} \Gamma(2 / 3)^{2} \Gamma(5 / 6)^{2}}$ & $\frac{(\sqrt{2}-1)^{-1} \pi}{2^{3 / 2} \Gamma(5 / 8)^{2} \Gamma(7 / 8)^{2}}$ \\
\hline
\end{tabular}

we obtain $\theta_{0}(i)^{4}$ and $\theta_{2}(i)^{4}$. At $z=\rho_{1}=e^{\pi i / 3}$, by using the equation

${ }_{2} F_{1}\left(a+\frac{1}{3}, 3 a ; 2 a+\frac{2}{3} ; e^{\pi i / 3}\right)=2 \pi e^{\pi i a / 2} 3^{-(3 a+1) / 2} \frac{\Gamma(2 a+2 / 3)}{\Gamma(a+1 / 3) \Gamma(a+2 / 3) \Gamma(2 / 3)}$

(see [EMOT, §2.9, equation (55), p. 105]) with $a=\frac{1}{6}$, we have

$$
\theta_{3}\left(\rho_{1}\right)^{4}={ }_{2} F_{1}\left(\frac{1}{2}, \frac{1}{2} ; 1 ; e^{\pi i / 3}\right)^{2}=\frac{2^{2} 3^{-3 / 2} \pi e^{\pi i / 6}}{\Gamma(2 / 3)^{2} \Gamma(5 / 6)^{2}} .
$$

Since

$$
\frac{\theta_{2}\left(\rho_{1}\right)^{4}}{\theta_{3}\left(\rho_{1}\right)^{4}}=\lambda^{b}\left(\rho_{1}\right)=\rho_{1} \quad \text { and } \quad \frac{\theta_{0}\left(\rho_{1}\right)^{4}}{\theta_{3}\left(\rho_{1}\right)^{4}}=1-\lambda^{b}\left(\rho_{1}\right)=e^{5 \pi i / 3},
$$

we obtain $\theta_{0}\left(\rho_{1}\right)^{4}$ and $\theta_{2}\left(\rho_{1}\right)^{4}$. At $z=\rho_{2}=e^{2 \pi i / 3}$, by using the values of $\theta_{0}\left(\rho_{1}\right)^{4}$, $\theta_{2}\left(\rho_{1}\right)^{4}$ and $\theta_{3}\left(\rho_{1}\right)^{4}$, and the property of elliptic theta functions

$$
\theta_{0}(z)=\theta_{3}(1+z), \quad e^{\pi i / 4} \theta_{2}(z)=\theta_{2}(1+z) \quad \text { and } \quad \theta_{3}(z)=\theta_{0}(1+z),
$$

we obtain $\theta_{0}\left(\rho_{2}\right)^{4}, \theta_{2}\left(\rho_{2}\right)^{4}$ and $\theta_{3}\left(\rho_{2}\right)^{4}$. At $z=\sqrt{2} i$, by using the equation

$$
{ }_{2} F_{1}(a, b ; a-b+1 ; z)=(1-z)^{-a}{ }_{2} F_{1}\left(\frac{a}{2}, \frac{a+1-2 b}{2} ; a-b+1 ;-\frac{4 z}{(1-z)^{2}}\right)
$$

(see [EMOT, §2.11, equation (32), p. 113]) with $a=b=\frac{1}{2}$ and the equation

$$
{ }_{2} F_{1}(a, b ; 1+a-b ;-1)=2^{-a} \frac{\Gamma(1+a-b) \Gamma(1 / 2)}{\Gamma(1-b+a / 2) \Gamma(1 / 2+a / 2)}
$$


TABLE A.2. Special values of $E_{4}$ and $E_{6}$.

\begin{tabular}{cccc}
\hline & $z=i$ & $z=\rho_{1}$ or $\rho_{2}$ & $z=\sqrt{2} i$ \\
\hline$E_{4}(z)$ & $\frac{3}{2^{6}} \frac{\Gamma(1 / 4)^{8}}{\pi^{6}}$ & 0 & $\frac{5}{2^{3}} \frac{\pi^{2}}{\Gamma(5 / 8)^{4} \Gamma(7 / 8)^{4}}$ \\
$E_{6}(z)$ & 0 & $\frac{2^{5}}{3^{3}} \frac{\pi^{3}}{\Gamma(2 / 3)^{6} \Gamma(5 / 6)^{6}}$ & $\frac{7}{2^{4}} \frac{\pi^{3}}{\Gamma(5 / 8)^{6} \Gamma(7 / 8)^{6}}$ \\
\hline
\end{tabular}

(see [EMOT, §2.8, equation (47), p. 104]) with $a=b=\frac{1}{4}$, we have

$$
\begin{aligned}
\theta_{3}(\sqrt{2} i)^{4} & ={ }_{2} F_{1}\left(\frac{1}{2}, \frac{1}{2} ; 1 ; \lambda^{\mathrm{b}}(\sqrt{2} i)\right)^{2}={ }_{2} F_{1}\left(\frac{1}{2}, \frac{1}{2} ; 1 ; 3-2 \sqrt{2}\right)^{2} \\
& =2^{-1}(\sqrt{2}-1)^{-1}{ }_{2} F_{1}\left(\frac{1}{4}, \frac{1}{4} ; 1 ;-1\right)^{2}=\frac{2^{-3 / 2}(\sqrt{2}-1)^{-1} \pi}{\Gamma(5 / 8)^{2} \Gamma(7 / 8)^{2}} .
\end{aligned}
$$

Since

$\frac{\theta_{2}(\sqrt{2} i)^{4}}{\theta_{3}(\sqrt{2} i)^{4}}=\lambda^{b}(\sqrt{2} i)=(\sqrt{2}-1)^{2} \quad$ and $\quad \frac{\theta_{0}(\sqrt{2} i)^{4}}{\theta_{3}(\sqrt{2} i)^{4}}=1-\lambda^{\mathrm{b}}(\sqrt{2} i)=2(\sqrt{2}-1)$,

we obtain $\theta_{0}(\sqrt{2} i)^{4}$ and $\theta_{2}(\sqrt{2} i)^{4}$.

\section{A.2. Eisenstein series}

Proposition A.2. Values at $z=i, \rho_{1}, \rho_{2}$ and $\sqrt{2} i$ of Eisenstein series $E_{4}(z)$ and $E_{6}(z)$ are given in Table A.2.

Proof. Let $\tau \in \mathbb{H}, L=\mathbb{Z} \oplus \mathbb{Z} \tau$ and let $\wp(z)$ be the Wierstrass $\wp$ function with the lattice $L . g_{2}(\tau)$ and $g_{3}(\tau)$ are defined by the equation

$$
\wp^{\prime}(z)^{2}=4 \wp(z)^{3}-g_{2}(\tau) \wp(z)+g_{3}(\tau) .
$$

By the well-known facts $g_{2}(\tau)=\frac{4}{3} \pi^{4} E_{4}(\tau)$ and $g_{3}(\tau)=\frac{8}{27} \pi^{6} E_{6}(\tau)$, we have

$$
\Delta(\tau)=g_{2}(\tau)^{3}-27 g_{3}(\tau)^{2}=\frac{2^{6}}{3^{3}} \pi^{12}\left(E_{4}(\tau)^{3}-E_{6}(\tau)^{2}\right) .
$$


We also have the equation

$$
\begin{aligned}
\Delta(\tau) & =(2 \pi)^{12} e^{2 \pi i \tau} \prod_{n=1}^{\infty}\left(1-e^{\pi i n \tau}\right)^{24}=(2 \pi)^{4} \theta_{1}^{\prime}(\tau)^{8} \\
& =2^{4} \pi^{12}\left(\theta_{0}(\tau) \theta_{2}(\tau) \theta_{3}(\tau)\right)^{8}=2^{4} \pi^{12} \theta_{2}(\tau)^{8} \theta_{3}(\tau)^{8}\left(\theta_{3}(\tau)^{4}-\theta_{2}(\tau)^{4}\right)^{2} .
\end{aligned}
$$

By the above two expressions we obtain

$$
\frac{2^{6}}{3^{3}} \pi^{12}\left(E_{4}(\tau)^{3}-E_{6}(\tau)^{2}\right)=2^{4} \pi^{12}\left(\theta_{0}(\tau) \theta_{2}(\tau) \theta_{3}(\tau)\right)^{8} .
$$

On the other hand, we have the well-known expression

$$
J(\tau)=P\left(\lambda^{b}(z)\right)=\frac{4}{27} \frac{\left(\theta_{2}(\tau)^{8}-\theta_{2}(\tau)^{4} \theta_{3}(\tau)^{4}+\theta_{3}(\tau)^{8}\right)^{3}}{\theta_{2}(\tau)^{8} \theta_{3}(\tau)^{8}\left(\theta_{3}(\tau)^{4}-\theta_{2}(\tau)^{4}\right)^{2}}=\frac{E_{4}(\tau)^{3}}{E_{4}(\tau)^{3}-E_{6}(\tau)^{2}} .
$$

Thus, we have

$$
\begin{gathered}
E_{4}(\tau)=\theta_{2}(\tau)^{8}-\theta_{2}(\tau)^{4} \theta_{3}(\tau)^{4}+\theta_{3}(\tau)^{8} \\
E_{6}(\tau)=\frac{1}{2}\left(\theta_{2}(\tau)^{4}-2 \theta_{3}(\tau)^{4}\right)\left(2 \theta_{2}(\tau)^{4}-\theta_{3}(\tau)^{4}\right)\left(\theta_{2}(\tau)^{4}+\theta_{3}(\tau)^{4}\right) .
\end{gathered}
$$

By using Proposition A.1, we obtain Proposition A.2.

\section{REFERENCES}

[EMOT] A. Erdélyi, W. Magnus, F. Oberhettinger and F. G. Tricomi. Higher Transcendental Functions. McGraw-Hill, New York, 1953.

[KY] M. Kaneko and M. Yoshida. The kappa function. Internat. J. Math. 14 (2003), 1003-1013.

[NS] N. Narumiya and H. Shiga. The mirror map for a family of $K 3$ surfaces induced from the simplest 3-dimensional reflexive polytope. Proc. Int. Conf. on Moonshine and Related Topics (Montréal, QC, 1999) (CRM Proc. Lecture Notes, 30). American Mathematical Society, Providence, RI, 2001, pp. 139-161.

Yuzen Tanaka, c/o Hironori Shiga Graduate School of Science and Technology Institute of Mathematics and Physics Chiba University 1-33 Yayoi-cyo Inage- $k u$ 263-8522 Chiba Japan

(E-mail:keith@hyper.ocn.ne.jp) 